\title{
DEBRIS FLUX COMPARISONS FROM THE GOLDSTONE RADAR, HAYSTACK RADAR, AND HAX RADAR PRIOR, DURING, AND AFTER THE LAST SOLAR MAXIMUM
}

\author{
C.L. Stokely ${ }^{1}$, E.G. Stansbery ${ }^{2}$, R.M. Goldstein ${ }^{3}$
}

(1) ESCG/Barrios Technology, Mail Code JE104, 2224 Bay Area Blvd., Houston, TX 77058, USA

(2) NASA, ARES, Mail Code KX, NASA JSC, Houston, TX 77058, USA

(3) Jet Propulsion Laboratory, California Institute of Technology, 4800 Oak Grove

Drive, Pasadena, CA 91109-8099, USA

The continual monitoring of low Earth orbit (LEO) debris environment using highly sensitive radars is essential for an accurate characterization of these dynamic populations. Debris populations are continually evolving since there are new debris sources, previously unrecognized debris sources, and debris loss mechanisms that are dependent on the dynamic space environment. Such radar data are used to supplement, update, and validate existing orbital debris models. NASA has been utilizing radar observations of the debris environment for over a decade from three complementary radars: the NASA JPL Goldstone radar, the MIT Lincoln Laboratory (MIT/LL) Long Range Imaging Radar (known as the Haystack radar), and the MIT/LL Haystack Auxiliary radar (HAX). All of these systems are highly sensitive radars that operate in a fixed staring mode to statistically sample orbital debris in the LEO environment. Each of these radars is ideally suited to measure debris within a specific size region. The Goldstone radar generally observes objects with sizes from $2 \mathrm{~mm}$ to $1 \mathrm{~cm}$. The Haystack radar generally measures from $5 \mathrm{~mm}$ to several meters. The HAX radar generally measures from $2 \mathrm{~cm}$ to several meters. These overlapping size regions allow a continuous measurement of cumulative debris flux versus diameter from $2 \mathrm{~mm}$ to several meters for a given altitude window. This is demonstrated for all three radars by comparing the debris flux versus diameter over $200 \mathrm{~km}$ altitude windows for 3 nonconsecutive years from 1998 through 2003. These years correspond to periods before, during, and after the peak of the last solar cycle. Comparing the year to year flux from Haystack for each of these altitude regions indicate statistically significant changes in subsets of the debris populations. Potential causes of these changes are discussed. These analysis results include error bars that represent statistical sampling errors, and are detailed in this paper.

\section{Introduction}

Increased solar activity is thought to play an important role in depleting the orbital debris population in low Earth orbits (LEO) through reentry by increased atmospheric drag. Simulation models of the effects of various levels of solar activity on the orbital debris environment indicate that high solar flux activity can dramatically affect orbits below about $600 \mathrm{~km}$. However, observation data of small debris ( $<10 \mathrm{~cm}$ diameter) before, during, and after a solar cycle peak have not been available until now. Radar data are used to supplement, update, and validate existing orbital debris models. Data relating the effects of solar activity to the behavior of the debris environment may be useful to further modeling efforts. This paper presents data during low solar activity in 1998, high solar activity in 2001, and low solar activity in 2003.

The atmospheric drag experienced by an orbiting object is a function of atmospheric density. Variations in atmospheric density are primarily driven by solar activity fluctuations. To give an indication of solar activity levels during the periods of interest, solar flux during the last two solar activity peaks is shown in figure 1 [1]. The ordinate is expressed in solar flux units (sfu, one solar flux unit $=10^{-22} \mathrm{~W} / \mathrm{m}^{2} / \mathrm{Hz}$ ). The observation periods are expressed in fiscal years. A fiscal year (FY) is defined as starting in October and ending in the following September; for example, fiscal year 2003 start in October 2002 and ends in September 2003. 


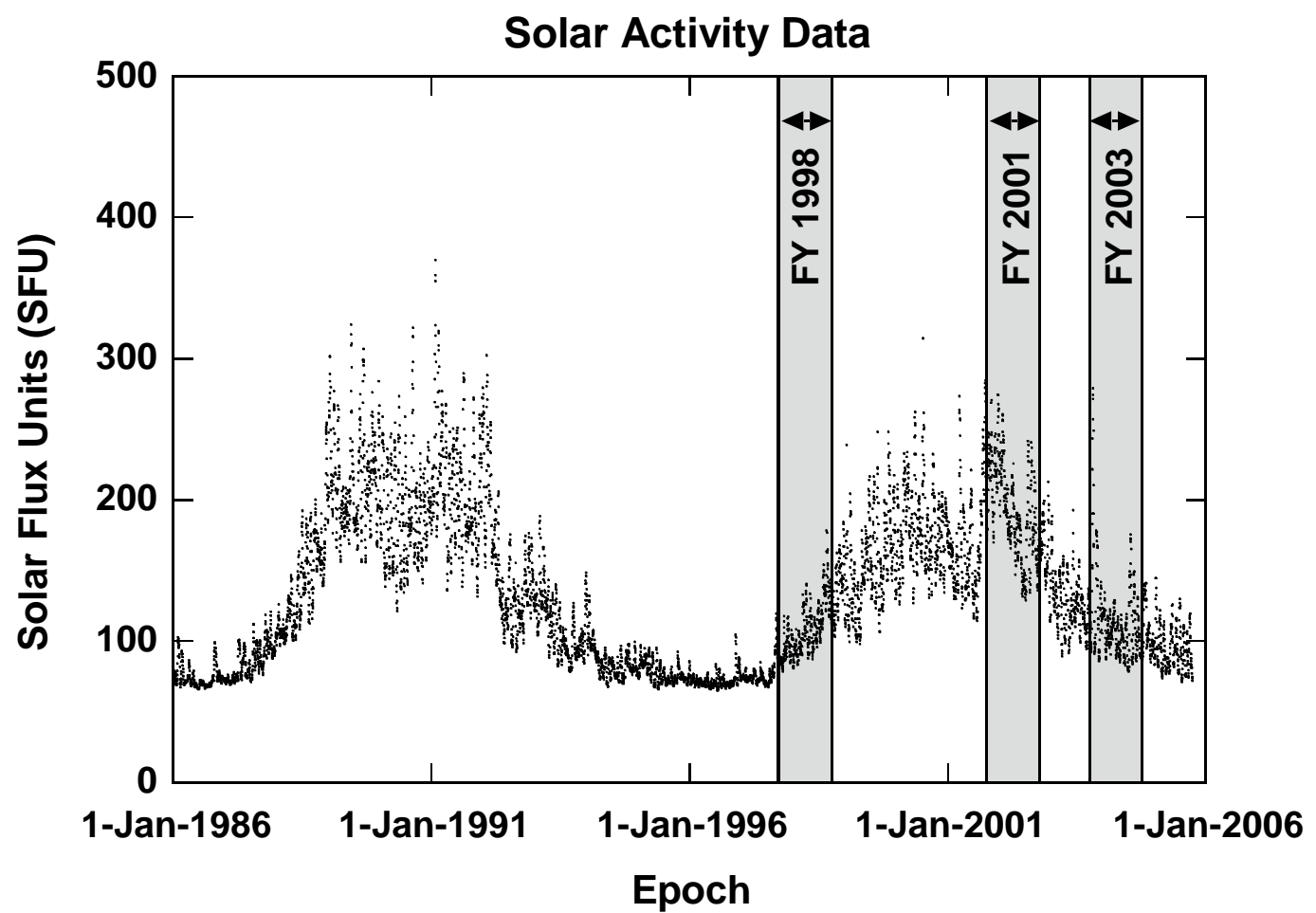

Figure 1. The solar flux activity versus year over the last two solar activity peaks. The highlighted zones on the figure indicate the time regions discussed in this paper.

\section{Radar Observations}

Only recently have radars been used to observe the orbital debris environment below 10 $\mathrm{cm}$. Highly sensitive radars are required for orbital debris observations since the observation time for a single debris piece is generally limited to less than 1 second and most debris is small $(<10$ $\mathrm{cm}$ ), returning radar signals with low signal-to-noise ratio (SNR). To obtain debris data down to 2 $\mathrm{mm}$ in diameter, NASA has concentrated its debris observation efforts on using the NASA JPL Goldstone radar, the MIT Lincoln Laboratory (MIT/LL) Haystack radar, and the MIT/LL Haystack Auxiliary radar (HAX). The overlapping size regions accessible to the radars allow comparisons in order to understand systematic and statistical variations in the data. Goldstone started intermittent orbital debris observations in the early 1990s; Haystack started observations in 1990; HAX started in 1994. The beginning of these observations was either at the peak or during the decline of solar activity. There are no significant radar data for debris with size less than $10 \mathrm{~cm}$ prior to the 1990 to 1991 solar activity maximum.

For debris observations, the radars are operated in a staring, or "beam park," mode in which the antenna is pointed at a specified elevation and azimuth and remains there while debris objects randomly pass through the field of view. The risk analysis associated with orbital debris generally starts with cumulative debris fluxes. This staring mode provides a fixed detection area important to the measurement of the debris flux, or number of objects detected per unit area per unit time. The fixed detection area is the conic frustum surface area of a cone with angular divergence determined by using the half-power $(3 \mathrm{~dB})$ point of the radars. The cumulative conic frustum flux quoted in this paper is referred to as the surface area flux, which relates to the cross sectional flux through the relation flux $_{c}=f_{l u x_{S}} \cdot \pi$.

The Haystack and HAX radars are circularly polarized radars with very high sensitivity. Both radars are colocated in Massachusetts at $42.62^{\circ}$ north latitude. To detect debris, Haystack and HAX use a pulsed continuous wave (CW) single frequency waveform. Haystack operates 
with a $3 \mathrm{~cm}$ wavelength and HAX operates with a $1.8 \mathrm{~cm}$ wavelength. Despite the HAX radar's shorter wavelength, its smaller diameter results in a larger radar detection volume than Haystack. HAX is not as sensitive as Haystack but its larger detection volume (via a larger beamwidth) allows it to collect increased statistics for larger debris objects. Haystack and HAX utilize a monopulse system that allows the position of a debris piece within the beam to be determined. The position of the debris in the beam is used to determine an accurate radar cross section (RCS), which is converted to size with an extension of the NASA Size Estimation Model (SEM) referred to as Statistical Size Estimation Model (SSEM) [2]. Both Haystack and HAX measure principal polarization and the orthogonal polarization radar returns. Both polarizations are required in order to use the SSEM, which provides statistical uncertainties in the observed populations. The error bars for the Haystack and HAX fluxes are statistical and assume Poisson sampling.

The Goldstone radar is an extremely sensitive bistatic system located in California's Mojave desert at $35.24^{\circ}$ north latitude. The presence of two dishes produces a complicated power gain beam pattern that is incorporated into the calculation of radar cross sections through the radar range equation. Using a $3.5 \mathrm{~cm}$ wavelength, the Goldstone radar transmits pairs of up and down chirps to determine the range and radial range rate. The transmitted pulses are circularly polarized. This system does not have a monopulse capability to determine where the debris passes relative to the center of the beam. This leads to inherent uncertainties of RCS measurements. There is no correction for this and therefore the quoted diameters are a lower limit since the particles do not necessarily go through the center of the beam. Additionally, since the Goldstone radar can measure only the principal polarization of the radar return signal, the measured RCS is slightly lower than the true RCS. This will result in the quoted diameters being slightly smaller than the true diameters. RCS data from the Goldstone radar is converted to size using the NASA Size Estimation Model (SEM) [2]. Errors are not given for the Goldstone radar since the systematic errors and biases are difficult to estimate.

The Haystack and HAX data discussed in this paper are for $75^{\circ}$ elevation with east pointing of the radars. The Goldstone radar is pointed near the zenith during debris observations. With these radar pointing conditions and the latitudes of the radars, the Goldstone radar observes orbits with inclinations between $35^{\circ}$ to $155^{\circ}$, and the Haystack and HAX radars observe orbits with inclinations between $43^{\circ}$ to $137^{\circ}$.

\section{Flux Measurements Year to Year}

Before comparing the fluxes over time from the influence of the solar activity, it is important to illustrate how the fluxes from the different radars compare at various altitudes. The altitudes discussed vary from $400 \mathrm{~km}$ to $1200 \mathrm{~km}$. Figures 2, 3, and 4 show the fluxes at $500 \mathrm{~km}$, $700 \mathrm{~km}, 900 \mathrm{~km}$, and $1100 \mathrm{~km}$ in $200 \mathrm{~km}$ bins for fiscal years 1998, 2001, and 2003 for the radars. There is no Goldstone data from 2002 to 2004. Therefore, a comparison is made between the FY2003 Haystack and HAX data and the Goldstone data from year 2005 . For comparison, the $10 \mathrm{~cm}$ catalogued population determined by the U.S. Space Surveillance Network is provided. The Space Surveillance Network can observe debris down to approximately $10 \mathrm{~cm}$ diameter. For the data shown, the Goldstone radar is pointed near the zenith. All the Haystack and HAX data are for the radar pointing at $75^{\circ}$ elevation, east pointing with the exception of the FY1998 HAX data; these data were collected with HAX pointed near the zenith. 



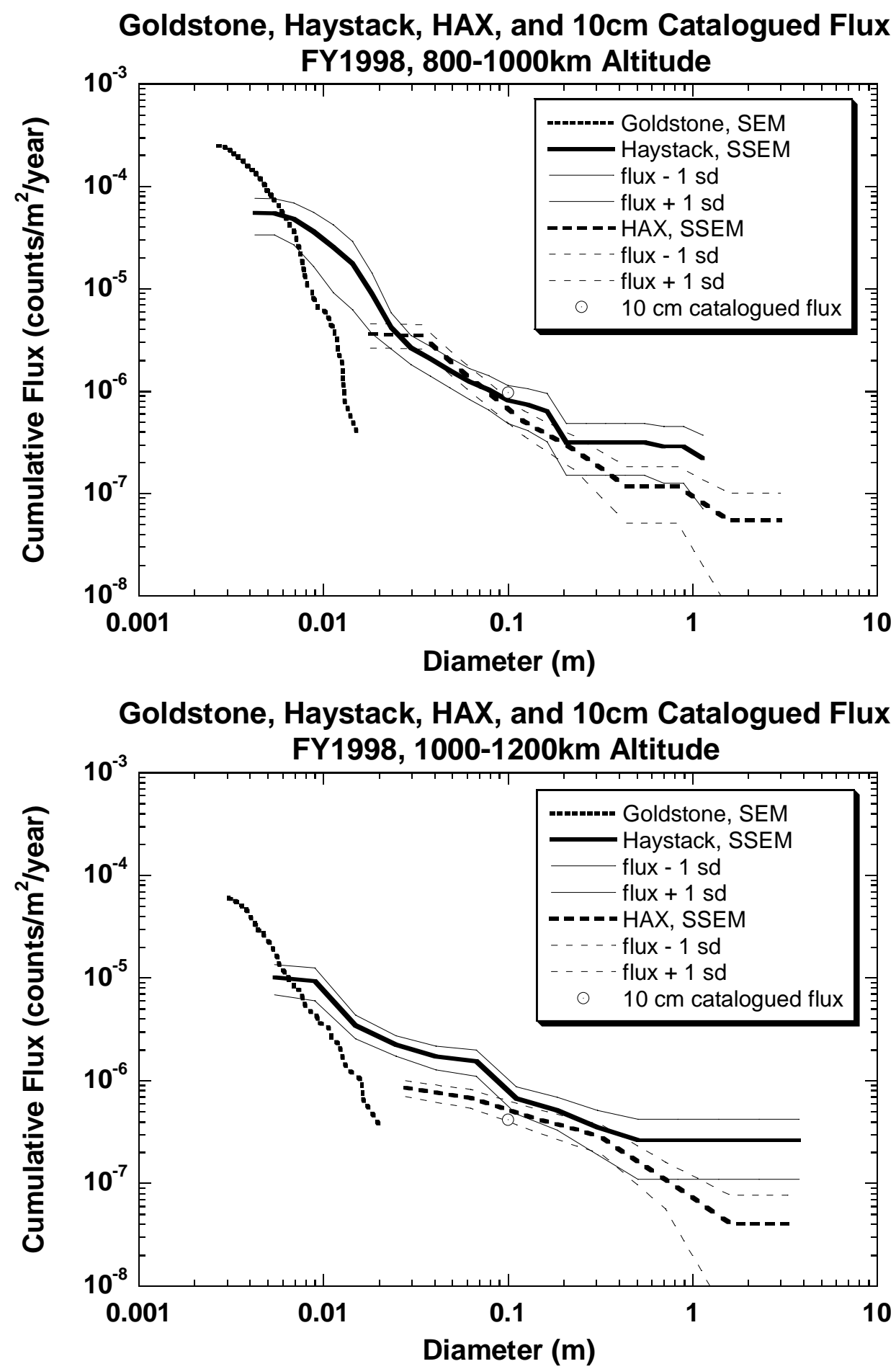

Figure 2. The debris flux versus diameter at $400 \mathrm{~km}$ to $600 \mathrm{~km}, 600 \mathrm{~km}$ to $800 \mathrm{~km}, 800 \mathrm{~km}$ to $1000 \mathrm{~km}$, and $1000 \mathrm{~km}$ to $1200 \mathrm{~km}$ for the Goldstone, Haystack, HAX radars during FY1998. 


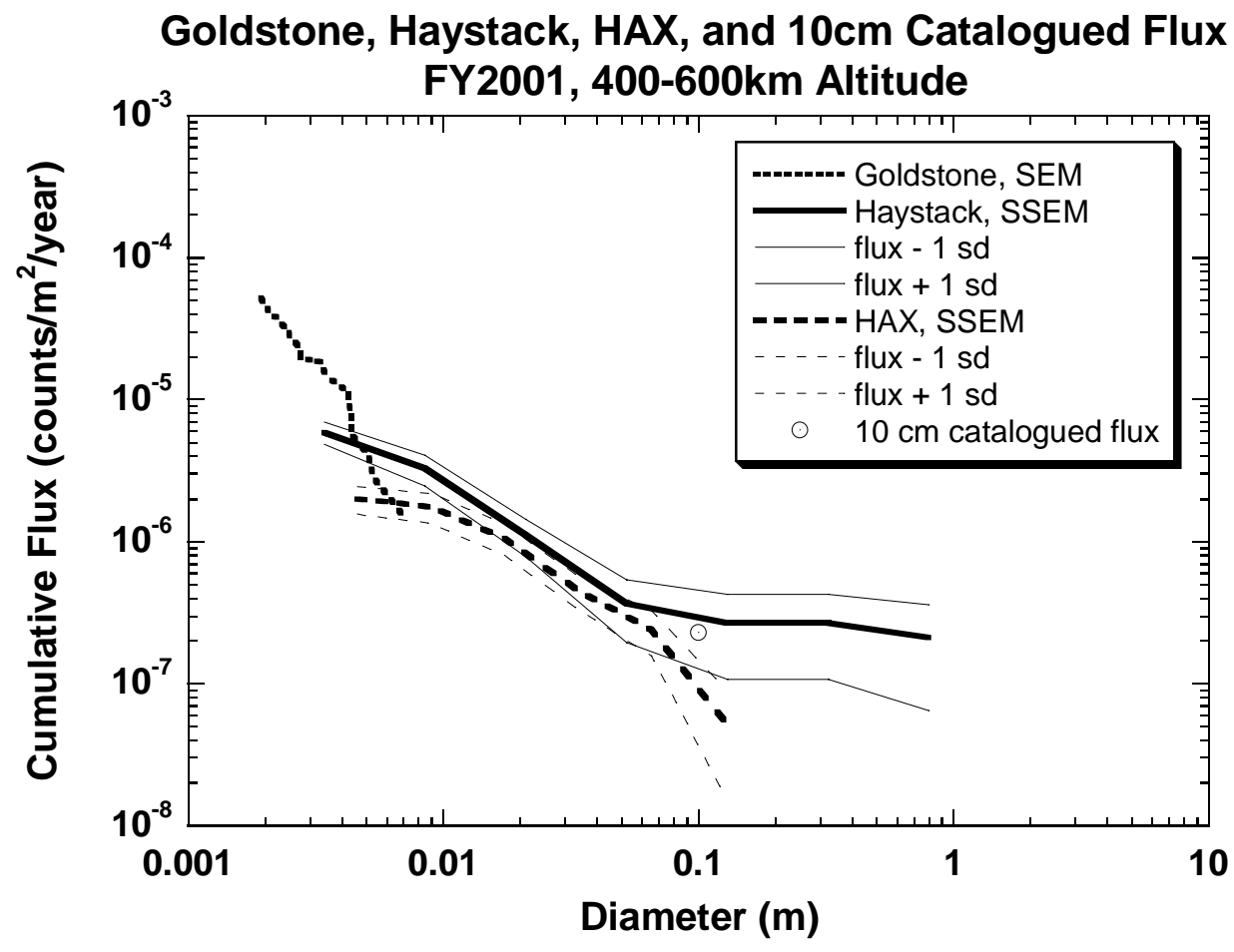

Goldstone, Haystack, HAX, and $10 \mathrm{~cm}$ Catalogued Flux

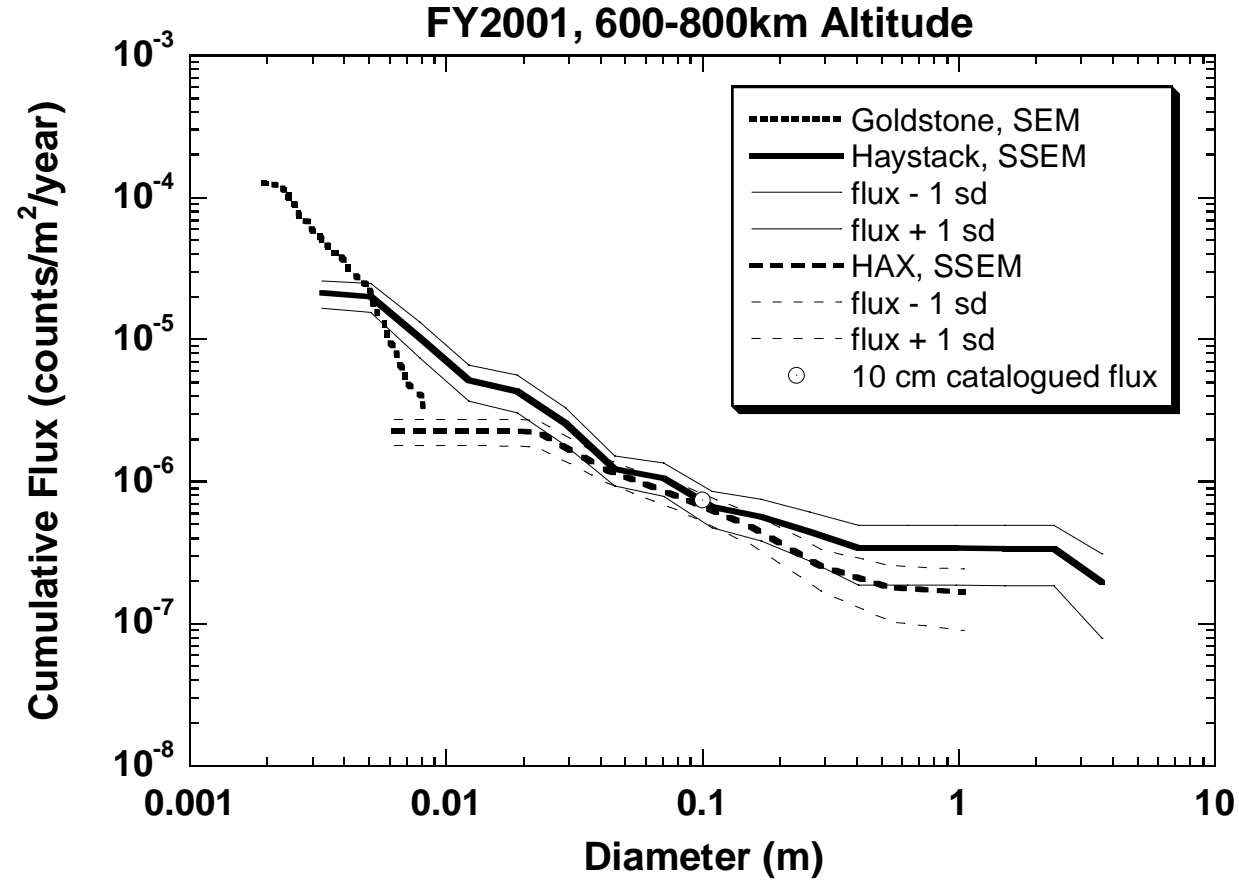




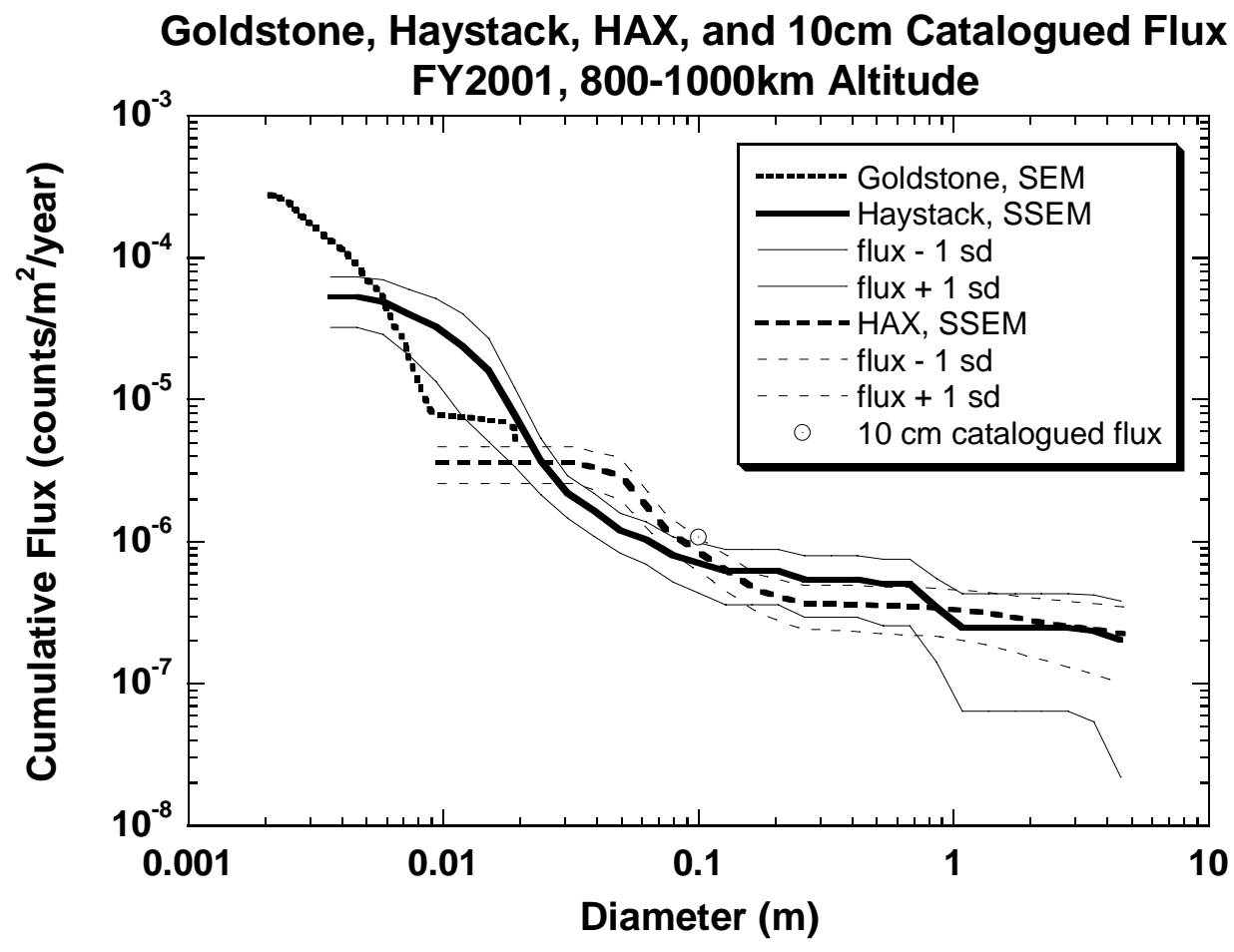

\section{Goldstone, Haystack, HAX, and $10 \mathrm{~cm}$ Catalogued Flux}

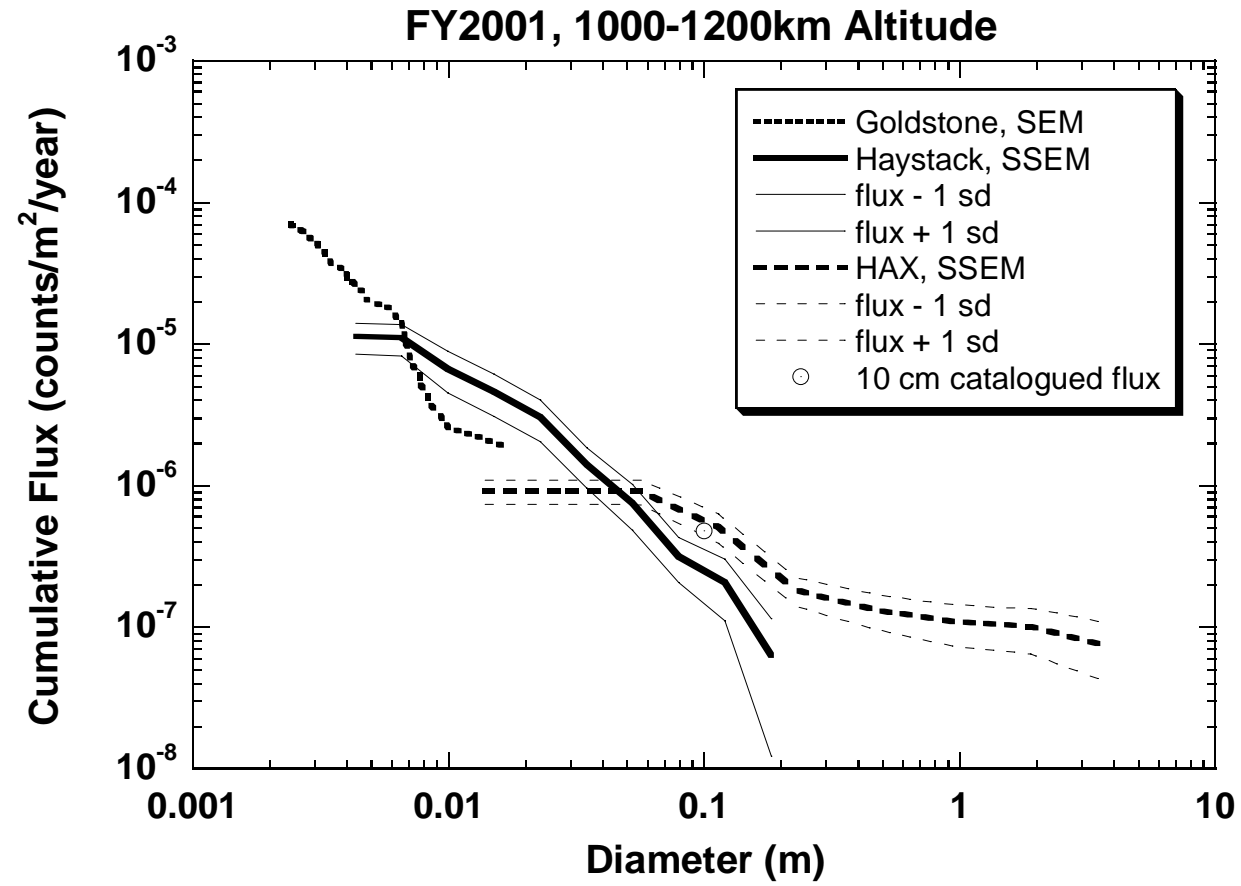

Figure 3. The debris flux versus diameter at $400 \mathrm{~km}$ to $600 \mathrm{~km}, 600 \mathrm{~km}$ to $800 \mathrm{~km}, 800 \mathrm{~km}$ to $1000 \mathrm{~km}$, and $1000 \mathrm{~km}$ to $1200 \mathrm{~km}$ for the Goldstone, Haystack, HAX radars during FY2001. 
Goldstone, Haystack, HAX, and $10 \mathrm{~cm}$ Catalogued Flux FY2003, 400-600km Altitude

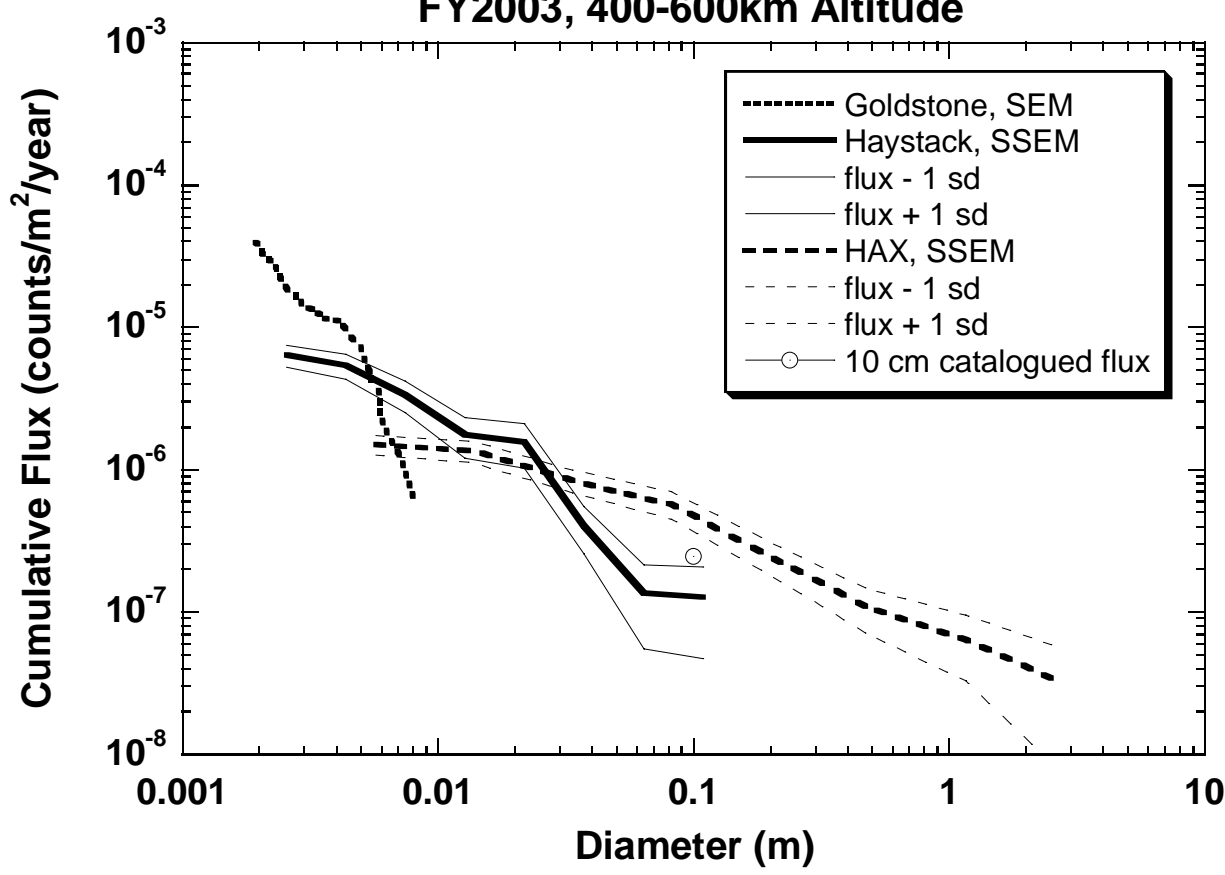

Goldstone, Haystack, HAX, and $10 \mathrm{~cm}$ Catalogued Flux

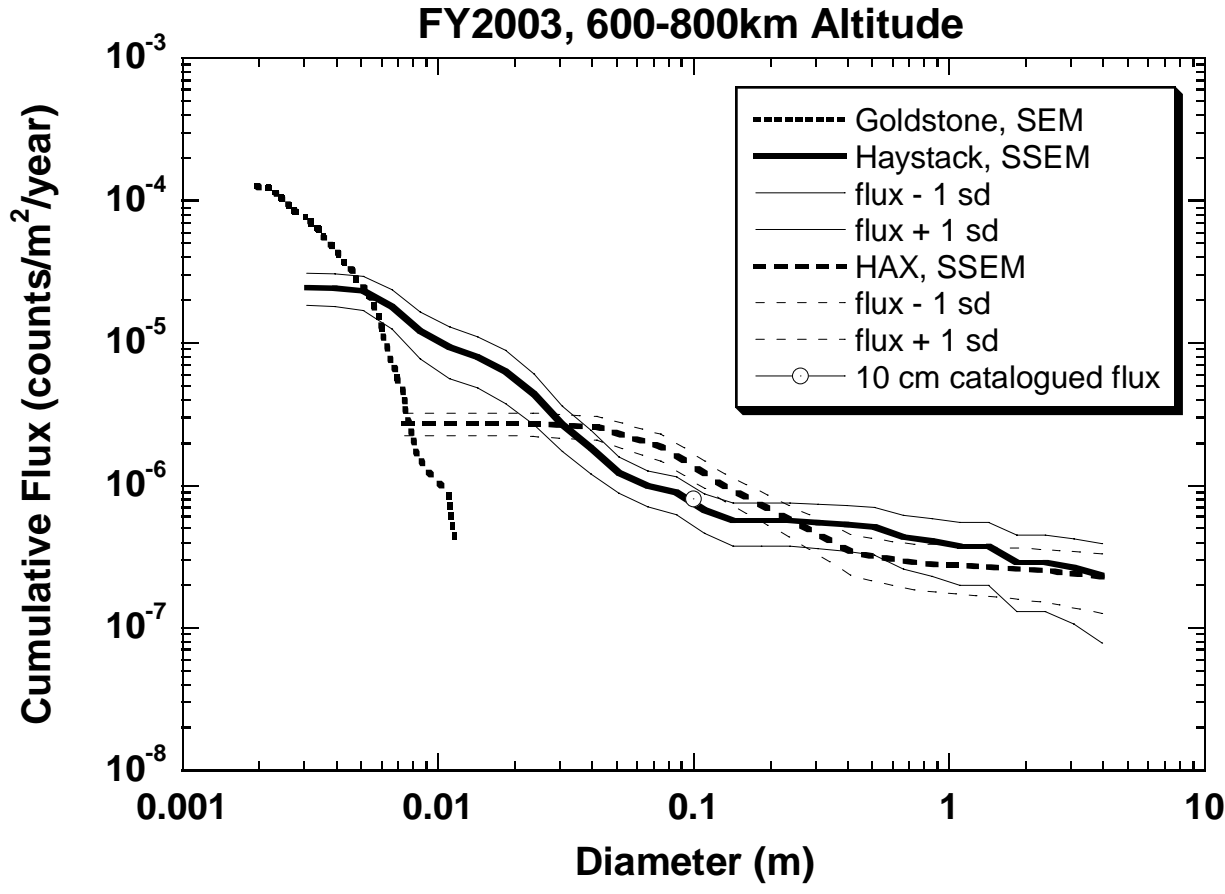



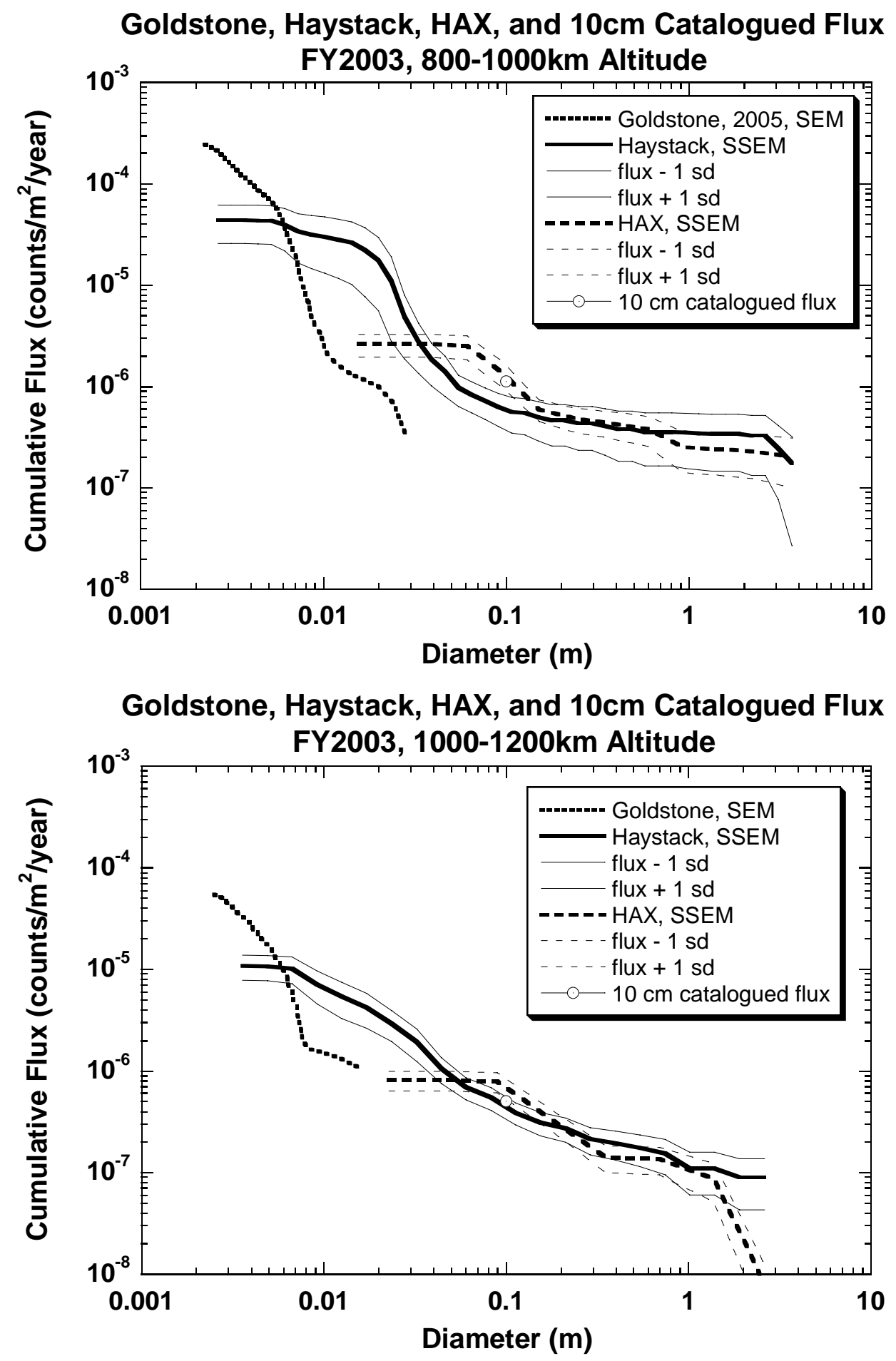

Figure 4. The debris flux versus diameter at $400 \mathrm{~km}$ to $600 \mathrm{~km}, 600 \mathrm{~km}$ to $800 \mathrm{~km}, 800 \mathrm{~km}$ to $1000 \mathrm{~km}$, and $1000 \mathrm{~km}$ to $1200 \mathrm{~km}$ for the Goldstone, Haystack, HAX radars during FY2003.

There are experimental caveats that must be realized when interpreting these radar data. As expected, all the radars show a roll-off in sensitivity at small sizes due to a decreased detection probability. The debris flux observed by the Goldstone radar abruptly drops above 8 $\mathrm{mm}$ compared to the Haystack data. This is a consequence of a signal saturation problem of the data acquisition system at the Goldstone radar, limiting the maximum size visible at a particular 
altitude [3]. Goldstone flux data for debris with diameters between $2 \mathrm{~mm}$ to $8 \mathrm{~mm}$ should be accurate even with its systematic limitations. In FY2003, malfunctions in the data acquisition system at the Haystack and HAX radars reduced the capability of the Haystack radar to detect debris less than $1 \mathrm{~cm}$, and the HAX radar to detect debris less than $4 \mathrm{~cm}$ [4]. This problem has since been remedied. Despite the limitations of the radars imposed by these data acquisition system problems, the excellent statistics for Haystack and HAX data during FY2003 have greatly helped in characterizing the small debris environment.

In figures 2, 3, and 4, there are some differences between Haystack and HAX for the largest sizes. Also evident are some slight differences in flux measurements to the $10 \mathrm{~cm}$ catalogued population. Assuming Poisson statistics may underestimate the errors since the debris environment can be very nonuniform. A debris swarm observed by the radar can significantly bias the flux measurement [5]. To demonstrate this, figure 5 illustrates the daily count rate versus the day of the year during Haystack observations near the beginning of FY2003. The error bars are derived assuming Poisson sampling errors. Some of the count rates are substantially different, even if 3-sigma error bars were considered. More accurate error estimates of the debris flux may require substantially more observation hours.

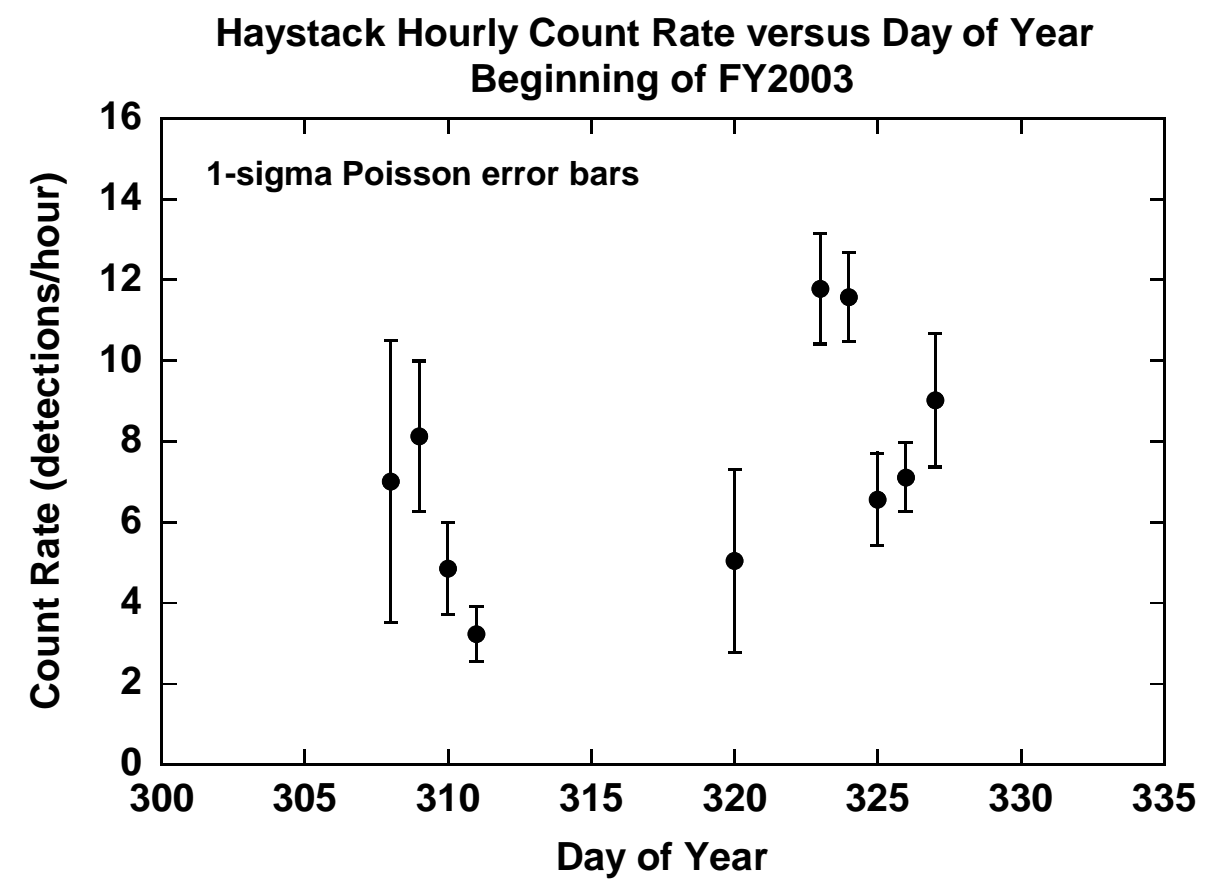

Figure 5. The count rate versus the day of the year for Haystack over a few days in the beginning of FY2003.

\section{Comparing Debris Flux Over the Years}

To restrict the scope of this work, only the year to year variations of the Haystack data are discussed. An analysis of the Haystack data from year to year indicate some changes in the average flux for the lower altitude bins among subsets of the debris population. Many of these changes typically vary from $10 \%$ to $30 \%$, consistent with some debris model estimates [1]. Even $10 \%$ to $30 \%$ variations in flux due to solar activity are considered significant but these changes cannot be distinguished from 1-sigma statistical variations, however. As seen in the figures, typical 1-sigma error estimates can range from $30 \%$ to $45 \%$ of the mean flux.

Figures $6,7,8$, and 9 show the year to year flux for altitudes $400 \mathrm{~km}$ to $600 \mathrm{~km}, 600 \mathrm{~km}$ to $800 \mathrm{~km}, 800 \mathrm{~km}$ to $1000 \mathrm{~km}$, and $1000 \mathrm{~km}$ to $1200 \mathrm{~km}$, respectively. The $400 \mathrm{~km}$ to $600 \mathrm{~km}$ 
altitude region illustrates a statistically significant decrease in the debris population below $1 \mathrm{~cm}$ before, during, and after the solar cycle maximum, as shown in figure 6 . For the smallest debris, the decline of the mean flux is more than $50 \%$.

The $600 \mathrm{~km}$ to $800 \mathrm{~km}$ region shows a statistically significant increase in the debris population above $3 \mathrm{~cm}$ before, during, and after the solar cycle maximum. For the largest debris, the mean flux increases by a factor of approximately 2 . In both figure 6 and figure 7, the changes in flux occur from 1998 to 2001, with almost no change from 2001 to 2003.

As shown in figure 8 , the $800 \mathrm{~km}$ to $1000 \mathrm{~km}$ regions shows no statistically significant variation in debris flux versus epoch. The $1000 \mathrm{~km}$ to $1200 \mathrm{~km}$ regions shown in figure 9 indicates some variations for larger sizes versus epoch but poor statistics at large sizes prohibit firm conclusions.

\section{Haystack Flux during FY1998, FY2001, and FY2003 400-600km Altitude}

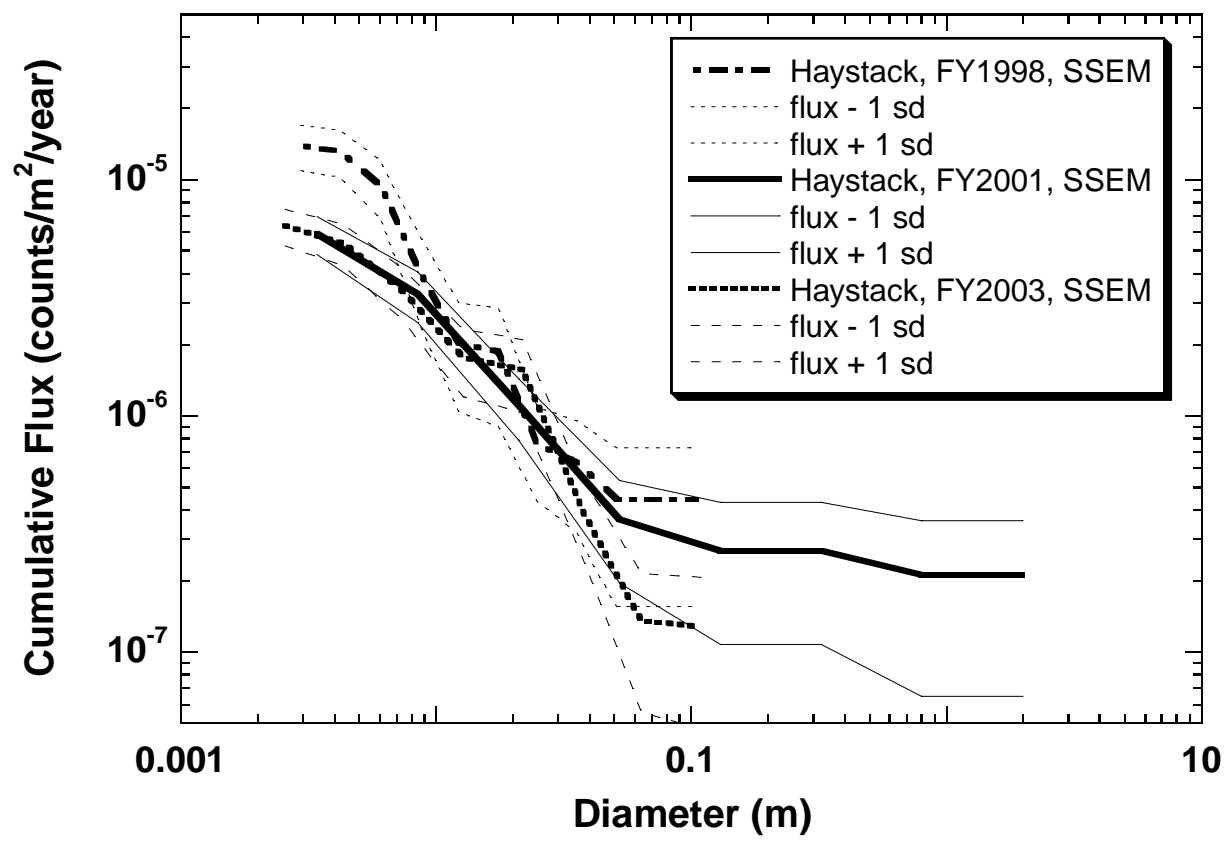

Figure 6. The debris flux versus diameter at $400 \mathrm{~km}$ to $600 \mathrm{~km}$ for Haystack $75^{\circ}$ elevation, east pointing measurements during FY1998, 2001, and 2003. 


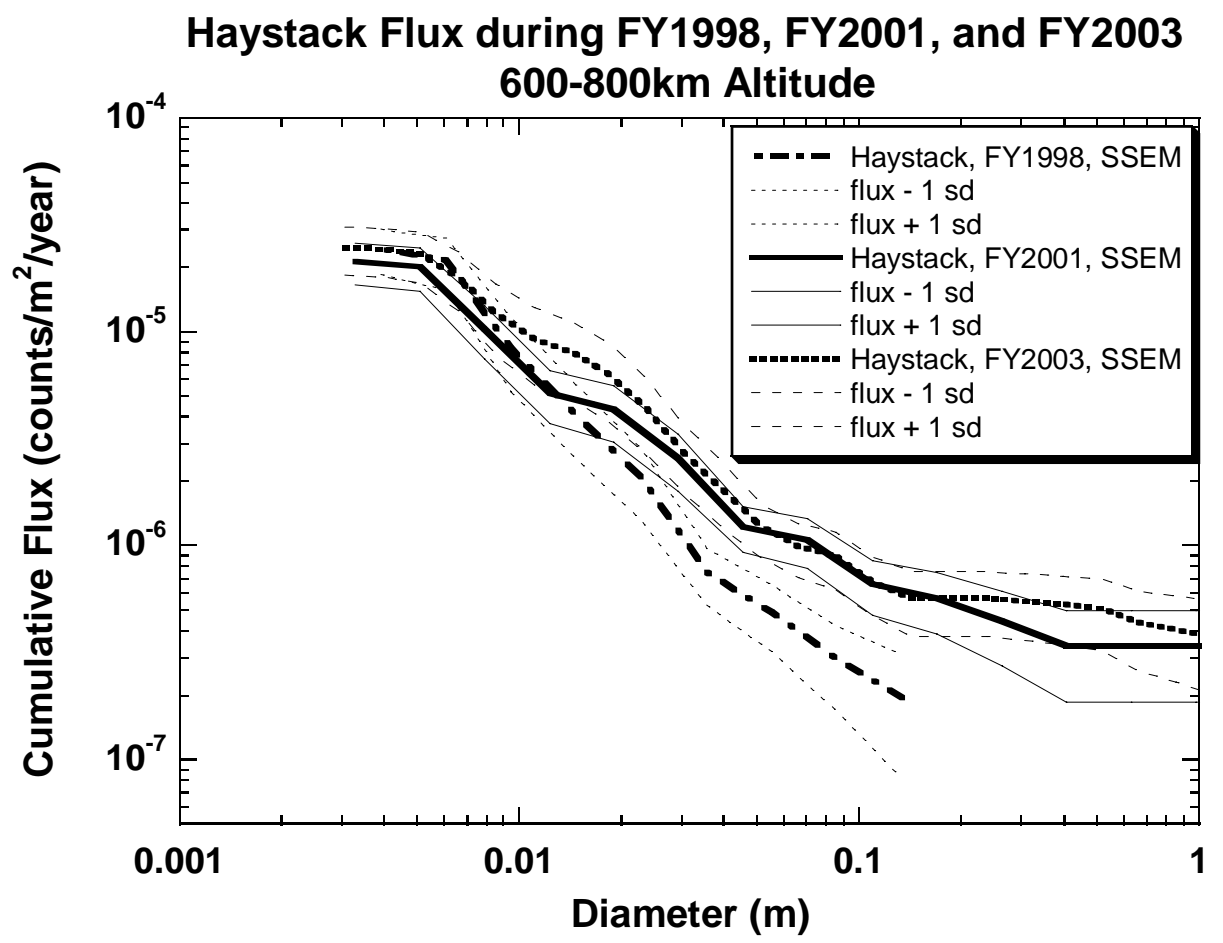

Figure 7. The debris flux versus diameter at $600 \mathrm{~km}$ to $800 \mathrm{~km}$ for Haystack $75^{\circ}$ elevation, east measurements during FY1998, 2001, and 2003.

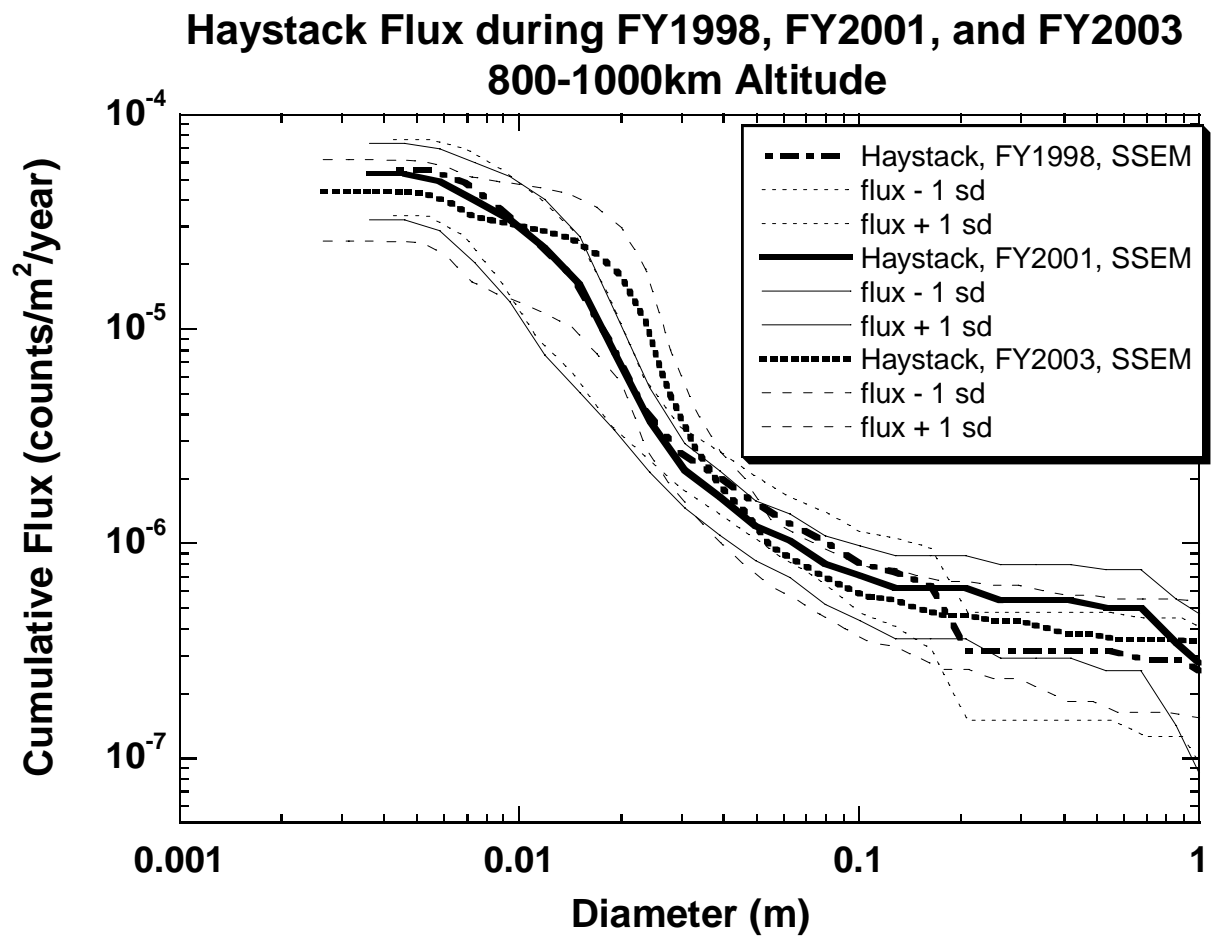

Figure 8. The debris flux versus diameter at $800 \mathrm{~km}$ to $1000 \mathrm{~km}$ for Haystack $75^{\circ}$ elevation, east measurements during FY1998, 2001, and 2003. 


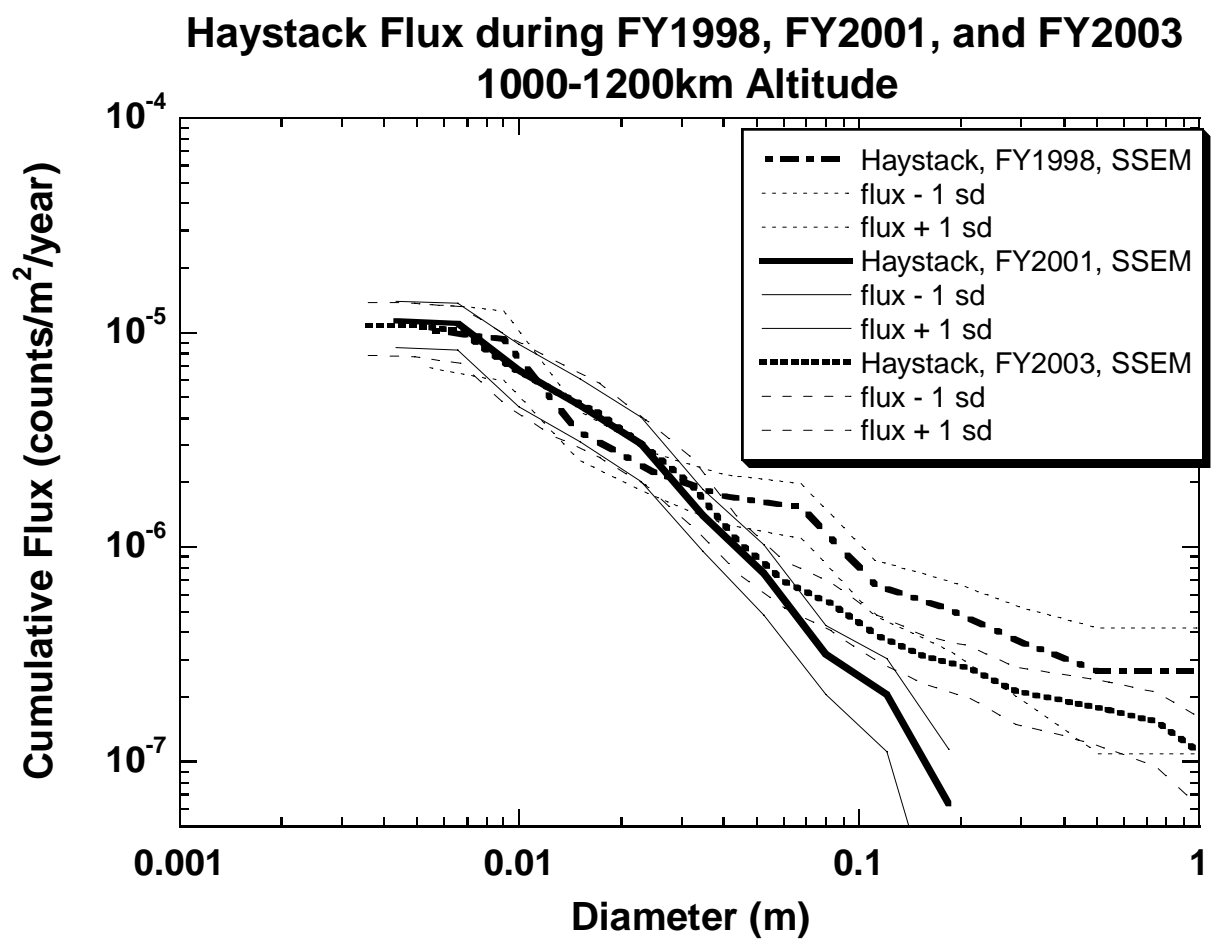

Figure 9. The debris flux versus diameter at $1000 \mathrm{~km}$ to $1200 \mathrm{~km}$ for Haystack $75^{\circ}$ elevation, east measurements during FY1998, 2001, and 2003.

Isolating the effects of solar activity from unknown debris sources is not completely possible. There are however some plausible simple scenarios that may explain the trends in the debris populations for each of the altitude windows. Consider that smaller debris have on average a larger area-to-mass ratio than larger debris, assuming similar shapes. For example, a sphere of radius $r$ and uniform density $\rho$ has an area-to-mass $=3 /(\rho \cdot r)$. Hence smaller debris should descend faster than larger debris due to the effects of atmospheric drag. This may explain the resultant decrease of the smallest debris for $400 \mathrm{~km}$ to $600 \mathrm{~km}$. The increase in the population of larger debris between $600 \mathrm{~km}$ to $800 \mathrm{~km}$ may simply be a result of new debris being generated in this region. If this is the case, the population of small debris should also increase. The lack of increase of small debris may simply be the result of a reduction of the small debris population from increased atmospheric drag via an increased solar flux mixed with an overall increase in the debris population from debris sources. Between $800 \mathrm{~km}$ to $1200 \mathrm{~km}$, the atmospheric drag is expected to be much smaller than at lower altitudes, so changes are most likely the result of new debris sources, collisions among existing debris, or statistical errors from inadequate observation periods.

\section{The RORSAT NaK Population}

It is important to realize that the space debris populations can be highly dynamic. Unequivocally separating the effects on the debris population from an increased solar flux in an environment with unknown debris sourcing is not completely possible. However, of special interest is the large population of small spherical droplets of eutectic sodium-potassium (NaK) between $800 \mathrm{~km}$ to $1000 \mathrm{~km}$ altitude in an inclination band centered near $65^{\circ}$ inclination. This population was identified in 1995 as metallic coolant leaked from fast neutron reactors that separated from the Russian Radar Ocean Reconnaissance SATellites (RORSATs) at the end of their useful lifetime [6]. This debris is easily identifiable using altitude, inclination, and RCS polarization data. Additionally, there are no new sources of NaK in this region, so effects of the 
solar activity can be illustrated. The median altitude of the NaK population (in near circular orbits) is seen to decrease noticeably during the onset of increased solar activity in 1998 . This is illustrated in figure 8. For comparison, a smoothed (15 point sliding average) solar activity curve versus epoch is also shown in figure 10.

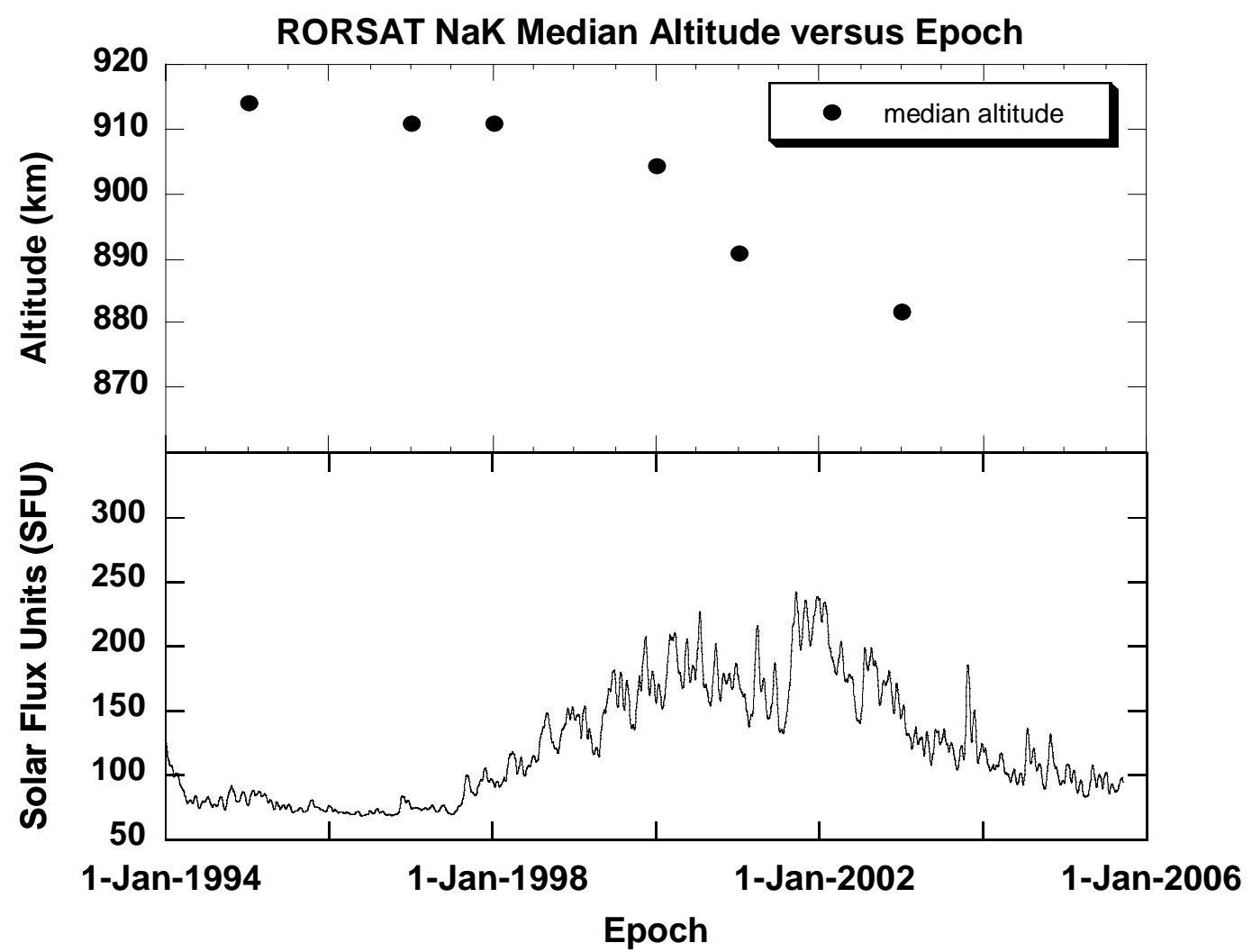

Figure 10. The median altitude of NaK versus year. A smoothed solar activity curve versus year is also shown for comparison.

The average size of the spherically conducting NaK debris is near the wavelength of the debris observation radars, resulting in Mie resonances. With Mie resonances, a RCS measurement can equate up to 3 noticeably different sizes [7]. This complicates the estimation of a reliable size distribution for this population. With a known size distribution of spheres with well known physical properties, the rate of fall of the NaK population provides a direct measurement of atmospheric drag at $800 \mathrm{~km}$ to $1000 \mathrm{~km}$ resulting from solar activity, and is a direction for future research.

\section{Conclusions}

The very large coverage in debris sizes allowed by utilizing the three complementary radars is vital for assessing and understanding the orbital debris populations, especially with the influence of solar activity. Statistical errors prohibit firm conclusions of the effects of increased solar activity on the debris populations, except for debris smaller than $1 \mathrm{~cm}$ diameter in the vicinity of $500 \mathrm{~km}$ altitude and debris larger than $3 \mathrm{~cm}$ near $700 \mathrm{~km}$ altitude. Some of the count rates measured by the radars vary substantially due to the highly inhomogeneous debris populations. Providing accurate error estimates of debris flux may require substantially more radar observation hours and could help isolate year to year changes in the debris populations. It is difficult to absolutely determine the effects of increased solar activity since there are uncontrolled and unknown debris sources. A good benchmark for studying the effects of solar activity are the RORSAT NaK spherical droplets since they are easily identifiable, have well known physical properties, and 
there are no new sources of RORSAT NaK debris. Studying this population may lead to a better understanding of the atmosphere near $900 \mathrm{~km}$ and its relation to solar variations.

\section{References}

1. Whitlock, D., Modeling the Effect of High Solar Activity on the Orbital Debris Environment. Orbital Debris Quarterly News, 10, 2, pp. 4-5, April, 2006.

2. Xu, Y.-I and C.L. Stokely. A Statistical Size Estimation Model for Haystack and HAX Radar Detections, $56^{\text {th }}$ International Astronautical Congress, Fukuoka, Japan, 2005.

3. Matney, M.J., et al. Recent Results from the Goldstone Radar, Adv. Space Res., 23, No. 1, p5-12,1999.

4. Stokely, C.L., et al. Haystack and HAX Radar Measurements of the Orbital Debris Environment; 2003, NASA/JSC Publication JSC-62815, Houston, TX, to be published.

5. Barton, D. K., et al., Final Report of the Haystack Orbital Debris Data Review Panel, NASA/JSC Technical Memorandum 4809, Houston, TX, February 1998.

6. Kessler, D. J., et al. A Search for a Previously Unknown Source of Orbital Debris: The Possibility of a Coolant Leak in Radar Ocean Reconnaissance Satellites. JSC27737 February 1997.

7. Knott, E.F., et al. Radar Cross Section, Artech House, Boston, MA, 1993. 